\title{
Les vies ultérieures de Tchicaya U Tam'Si
}

Post-scriptum à « Tchicaya passion »

\section{Nicolas Martin-Granel}

\section{(2) OpenEdition}

1 Journals

Édition électronique

URL : http://journals.openedition.org/coma/3753

DOI : 10.4000/coma.3753

ISSN : 2275-1742

\section{Éditeur}

Institut des textes \& manuscrits modernes (ITEM)

\section{Référence électronique}

Nicolas Martin-Granel, «Les vies ultérieures de Tchicaya U Tam'Si », Continents manuscrits [En ligne], 4 | 2015, mis en ligne le 20 février 2019, consulté le 16 avril 2021. URL : http:// journals.openedition.org/coma/3753; DOI : https://doi.org/10.4000/coma.3753

Ce document a été généré automatiquement le 16 avril 2021

\section{(c) $(1) \odot$}

Continents manuscrits - Génétique des textes littéraires - Afrique, Caraîbe, dispora est mis à disposition selon les termes de la licence Creative Commons Attribution - Pas d'Utilisation Commerciale - Pas de Modification 4.0 International. 


\title{
Les vies ultérieures de Tchicaya U Tam'Si
}

\author{
Post-scriptum à « Tchicaya passion »
}

Nicolas Martin-Granel

1 "Tchicaya passion ${ }^{1}$ ", ce numéro d'hommage à Tchicaya persiste et signe (par quatre signatures de plus) : l'écrivain y est sacré prophète à titre posthume, la première de ses vies ultérieures, la plus communément admise, on l'a $\mathrm{vu}^{2}$, dans la république des lettres congolaises. Ainsi, le duo, trio ou quarteron des fameux vers, "Un jour il faudra se prendre / marcher haut les vents / comme les feuilles des arbres... », continue sur sa lancée triomphale, avec pas moins de quatre mentions supplémentaires qui confirment la fortune de la réception prophétique du poète. Une telle réception se retrouve non seulement chez les frères ou fils cadets de la phratrie comme Alain Mabanckou ${ }^{3}$ ou Dieudonné Niangouna: "De toutes les bouches sonnaient "À travers vents et fleuves" ", mais aussi chez les éminents critiques " gaulois » qui ont connu l'homme. Jacques Chevrier se souvient d'une stèle au Maroc où les vers sont même gravés dans le marbre «en arabe et en français ${ }^{5}$ »; et pour Arlette Chemain, dans son témoignage, Tchicaya est forcément poète-prophète puisque « celui qui écrit: "J'enterre mes rêves qui se lèvent en semailles" ou encore "Un jour il faudra se prendre, marcher haut les vents", vers souvent cité par Henri Lopes, dès son premier recueil Le Mauvais Sang se voulait bâtisseur d'un avenir et à l'origine d'un monde meilleur. ${ }^{6}$ » À croire que tous les amis et lecteurs de Tchicaya, à l'instar d'obélix le gaulois, sont tombés dans la potion magique, que le syndrome du prophète court comme un feu de brousse, attisé par le vent du prophétisme avéré du poète.

2 Deuxième leçon à tirer, deuxième vie ultérieure toute tracée : père ou frère de Sony Labou Tansi. D'entrée de jeu, dès l'éditorial cosigné par Olivier Poivre d'Arvor, le directeur de Cultures France, et Boniface Mongo-Mboussa, un des coordinateurs ${ }^{7} \mathrm{du}$ numéro, il est considéré comme le « "fils" littéraire ». Un fils prodigue sans doute, mais qui, selon Tati-Loutard, jouerait son rôle à contre-emploi, le rôle d'un père qui va réintégrer au sein de la phratrie le fils perdu à la périphérie, égaré sur les rives de la Seine : 
Par la suite, avec le succès de Sony Labou Tansi, Tchicaya U Tam'Si a sans doute été séduit par ce qui se tramait à Brazzaville en matière de littérature. Et c'est donc à travers Sony qu'il a, d'une certaine manière, participé à la phratrie ${ }^{8}$.

En mécène, Tchicaya accueille sans jalousie son presque homonyme Marcel Sony, futur Labou Tansi, qu'il rencontre pour la première fois à Nice en 1981. Il se vexe si celui qui lui doit son pseudonyme, et qu'il reçoit, le quitte pour se rendre une journée chez Béti. Et c'est "Henri” qui calmera Tam'si en paroles?.

4 Or, c'est précisément cette vie ultérieure de Tchicaya menée par son " presqu'homonyme » que je me propose à présent de suivre d'un peu plus près, jusque dans ses croisements ambigus et ses malentendus improbables ${ }^{10}$.

\section{TUT et SLT : père ou frère ? jumeaux ou rivaux ?}

5 Pourtant, quand on l'entend de la bouche même de Sony Labou Tansi, le récit de cette première rencontre diffère très sensiblement : point de grief d'ingratitude, ni de colère, juste une dispute dans la cuisine, un assaut d'humble humanité. C'était ici même, devant les étudiants de Bayardelle, il y a vingt ans, soit l'année même de la disparition du frère aîné, que Sony racontait ce premier face à face en des termes qui font songer à une rencontre d'amoureux plutôt que de frères ennemis. Voici ces paroles que je retranscris verbatim :

Pourquoi vouloir être le plus grand écrivain? Ça sert à quoi ? S'il y a quelqu'un qui m'a appris cette chose, c'est aussi Tchicaya. On a commencé à correspondre jusqu'en 79 , et je suis parti à Nice où j'étais invité à un festival. J'arrive et en montant j'entends dans la salle quelqu'un qui parlait le lingala, mais un lingala qui n'était pas très clair pour moi qui ai un peu grandi au Zaïre, pour qui donc il y a la finesse du Zaïre : je me dis que ça ne doit pas être quelqu'un de la source. Alors je cherche qui c'est et il me dit «tu ne connais pas? c'est moi Tchicaya». Je dis « ah bon ». C'est tout, c'est vrai que c'était comme ça, comme un choc. Puis on est sorti dans Nice, on a passé une semaine ensemble, on est allé dans un restaurant cette nuit-là à Nice avec Med Hondo que vous devez connaître, on a discuté, on a parlé pendant une semaine, on était devenu comme un père et son enfant. après on est allé à Paris, il m'a emmené dans sa maison de campagne, on discutait et on parlait de tout avec le respect parce c'est important de respecter même un petit enfant, il faut le respecter même si tu es grand. Donc il y avait cette humanité importante qui était là, on se disputait pour faire la cuisine, puis la vaisselle, il voulait faire la vaisselle, on se disputait les assiettes... Tu ne trouves pas ça au Congo. Chez nous, non, on met les mains dans les poches pour que les gens d'en bas travaillent. Les gens, parce qu'ils ont un boy, ils le considèrent comme une chose mais c'est un homme ! Il y a donc cette humanité chez Gérald - que vous n'avez pas connue peutêtre à part son écriture - l'humanité de quelqu'un qui est capable de se disputer la vaisselle... C'est comme ça qu'on est devenus parents, on a l'a décidé d'ailleurs c'est rare que gens décident d'être parents - qu'on serait père et fils. Comme je l'ai fait avec Sylvain Bemba, on avait décidé qu'on serait Bantsimba et Banzouzi, on l'a décidé pour des raisons claires, ce n'est pas de la démagogie c'est simplement parce qu'on se sentait frères ${ }^{11}$.

6 De cette rencontre d'où est gommée toute vexation de part et d'autre, SLT tire une leçon d'humanité à l'usage de son public étudiant. Elle devient un apologue, un exemple édifiant qui doit servir de modèle à la jeunesse. Sans doute Sony s'est rêvé sur ce modèle de «grand écrivain ». Avant d'adopter le nom de plume qu'on lui connaît, il 
avait signé en 1969 une première pièce de théâtre, Monsieur Tout Court, sous le pseudonyme de Sony-Ciano Soyinka, en référence et révérence au grand auteur nigérian qui recevra bientôt le prix Nobel de littérature. Puis, suite à la rencontre épistolaire avec Tchicaya, il en adopte un autre, plus proche de son nouveau mentor. Car ils se trouvent sur la même longueur d'onde poétique, tous deux branchés sur Rimbaud :

Tu dois connaître Tchicaya U-Tamsi ? Je lui ai écrit, puis je lui ai envoyé un recueil Le Pays Intérieur. Il a fêté ça. Et il se bat à taper le texte pour chercher un éditeur. À en juger à quatre de ses lettres où il dit qu'on se fête et qu'on est frères, c'est un type séduisant. J'aime d'ailleurs ce qu'il écrit. Ç'a du ventre. Pas comme ces types qui écrivent comme des professeurs. Rimbaud n'écrivait pas comme un universitaire : en cela il est et restera éblouissant. Il n'écrivait même pas comme un musicien, il écrivait comme un sang, comme un cœur. C'est un exemple unique et ensorcelant dans la littérature française. Vache! Je parle comme un professeur. Je hais ça. Allez ce con de Rimbaud! Il avait de la parole, je crois ${ }^{12}$.

7 Bien sûr, le fait que la référence à Rimbaud est commune aux deux poètes congolais, qu'elle les rapproche dans la conscience d'être tous deux nés sous le signe du même "mauvais sang ", permet au jeune poète de se hisser quasi à égalité avec l'aîné déjà reconnu, inscrit dans la lignée rimbaldienne. Cependant, il faut noter que cette référence n'est pas seulement positive, mais aussi et surtout polémique. Pour que la fraternisation soit une opération réussie, il ne faut pas seulement écrire comme Rimbaud, mais contre. Contre, en l'occurrence, "les professeurs ", contre le modèle « universitaire». Et donc, au moins implicitement, contre l'école de la Négritude, et plus précisément Senghor, qui pour Sony représente cette écriture savante et intellectuelle. À l'opposé de ce modèle honni, Tchicaya incarne une poétique du «ventre ", l'anti-modèle de la poésie nègre, voire bantoue. C'est en effet par cet ethnonyme identitaire que Senghor aurait voulu enrôler Tchicaya, à son corps défendant, dans la négritude.

\section{Tchicaya, Sony et Rimbaud : I'impossible triangle}

Sony s'est longtemps cherché des ascendants précurseurs, des auteurs qui puissent se porter garants de la lignée des poètes prophètes dans laquelle il s'inscrit. Sony a certes beaucoup lu, mais ses lectures affleurent peu dans ses propres textes. Les citations y sont rares, mais toutes de poètes. Verlaine se trouve nommé une fois dans ses poésies de jeunesse, celui de Rimbaud deux fois dans la correspondance, et encore pour annoncer la même citation qu'il voudrait faire sienne :

Projets? Il faut en avoir plein la peau et le cœur. C'est un droit. Parce que, disait Rimbaud: «Nous ne sommes pas au monde.» Et je corrige, en disant preuve à

l'appui que « nous ne sommes pas qu'au monde $»^{13}$.

Corriger Rimbaud, n'est-ce pas déjà une façon de se poser comme un pair? Surtout quand on sait la place qu'occupe Rimbaud dans le panthéon personnel de Sony, la toute première :

Il y a une chose épouvantable - c'est que le plus grand poète français de tous les temps (Rimbaud) n'ait réussi à dire qu'une chose, une seule : « nous ne sommes pas au monde $»^{14}$. 
10 Cependant, suivre la voie rimbaldienne est un parcours semé d'embûches. Tout d'abord, le désir de dire à la manière et à la suite de Rimbaud se heurte très vite au mur $\mathrm{du}$ (re)nom érigé par l'institution littéraire :

Je crois qu'on ne choisit pas tout à fait. Il y a l'itinéraire : j'ai commencé par écrire des poèmes, comme tout le monde. C'est je crois le genre que j'aime le mieux. Mais je me suis aperçu d'une chose: on n'a pas plus le droit d'écrire des poèmes tant qu'on n'a pas été Senghor ou Césaire. Cette chose ne vient pas de moi, je l'ai entendue des éditeurs. À Paris, en 1973, des gens chez qui j'ai montré mes poèmes, et qui ont dit qu'ils étaient beaux, mais il leur fallait un nom. On ne peut pas entrer nu-pieds dans le monde de Rimbaud. Et comme je voulais absolument dialoguer, je me suis mis à écrire autre chose. En fait ce qui compte, ce n'est pas le genre, mais les choses qu'on a à dire ${ }^{15}$.

11 Ce va-nu-pieds de la poésie ira donc se faire un nom ailleurs que dans «le monde de Rimbaud ", dans un autre genre plus ouvert aux formes nouvelles et étrangères, dans un genre sans frontières. D'autant que pour rejoindre l'art d'un Rimbaud, il lui faudrait aussi prouver auprès des instances de validation littéraire qu'un « petit sauvageon » est capable d'art :

Nous savons tous comment l'on refusa (et on le fait encore) aux Africains et aux Indiens toute prérogative artistique, tout ce qu'ils ont fait dans ce domaine étant délié de la prétendue gratuité de l'art par une raison profonde, physique et métaphysique, comme si Shakespeare avait jamais été gratuit, gratuit Rimbaud, gratuits Beethoven et Léonard de Vinci. J'aimerais qu'on me prouve la gratuité idéologique d'un Michel-Ange ou d'un Titien. L'art, c'est d'abord art de vivre. Art de vivre, manière d'humaniser, qu'on le veuille ou non ${ }^{16}$.

12 En ce point, les poétiques des deux frères congolais se rejoignent volontiers sur la même postulation existentielle, entre "art de vivre » pour celui-ci, et «comment vivre" pour celui-là. Sur ce point, il y a complicité. Mais la rivalité surgit avec la question du champ littéraire. La distance se creuse entre le centre de légitimation (Paris) et la périphérie (Congo).

Pour Sony, en effet, le principal obstacle pour entrer dans une carrière poétique sur les traces de l'homme aux semelles de vent, ce serait que la place - la seule qui vaille : Paris - du disciple africain serait déjà prise, entièrement occupée par «l'ancêtre ", c'est-àdire par « Tchicaya-Rimbaud » :

L'enfance est interrompue par les vagabondages politiques du père dans les grands froids parisiens, froids physiques, froids humains, froids de l'âme... perverti par la petite arrogance négresse de se sentir blanc quelque part une fois qu'on a touché et goûté Paris. Mais pour Tchicaya-Rimbaud quelque part, les lycéens parisiens ont tout de suite une senteur maléfique et un goût d'embûche: on n'est pas petit broussard dans Paris pour rien peut-être fatalement pour être poète toujours parti, toujours partant. [...] Ne voyez pas que les remous de surface d'un grand affamé d'Afriques, de Kongo, et d'humanisme, Tchicaya U Tam'si n'est pas, ainsi qu'il l'a naïvement déclaré à cor et à cris, la petite feuille qui parle et nomme un pays innommé - Le pays innommé c'est lui - le prince qui court comme un grand fleuve, affamé de vérité, de pays, de fraternité, d'envie d'être vivant vif, entendez vivant cru et épidermique, haineux du mensonge sous tous ses ciels et sous toutes ses formes. C'est de cette matière-là que sont faits les princes de la pensée, carrure Rimbaud ou Michaux ${ }^{17}$.

14 Or, s'il est vrai que deux crocodiles ne peuvent cohabiter dans un marigot, a fortiori dans un grand fleuve comme le Congo, il ne saurait y avoir ensemble deux poètes de telle « carrure ». Sony ira à la recherche d'autres parrains poétiques, Artaud et surtout 
Hugo. Et réglera ses comptes avec Rimbaud dans un poème magnifique nommé précisément Lettre infernale ${ }^{18}$. Dont je doute que Tchicaya ait eu connaissance.

\section{TAM'SI et TANSI : à prophète, prophète et demi}

15 Faute d'être frères en poésie, unis par «le mauvais sang ", ils le seront en prophétie, réunis par le ventre. Ou du moins, c'est la vision du cadet pour qui l'aîné ne saurait se «disculper» de son prophétisme. Tchicaya refuse d'être enrôlé dans la lignée des prophètes dont Victor Hugo est devenu pour Sony le garant ancestral, la marque littéraire. D'où sans doute le souci de Tchicaya de se démarquer de son cadet: «On nous confond à cause de la fausse ressemblance de nos pseudonymes. »

De son côté, Sony prend lui aussi ses distances avec son modèle, allant jusqu'à récuser la filiation des pseudonymes d'écrivains, Tam'si et Tansi. En 1987, répondant à la question «quel rapport peut-on dresser entre ces deux noms», il (dé)nie toute influence :

Il y a un rapprochement entre le vili et le kongo qui sont deux dialectes du pays. Mais il n'y a pas de lien entre les deux noms, car «Tchicaya U Tamsi » est une phrase en vili qui signifie " Petite feuille qui parle pour son pays ", tandis que Sony Labou Tansi est un nom formé à partir de trois autres. Mon père était danseur. Ceux-ci portent en dansant une petite queue qu'on appelle « labou ». D'où son nom que j'ai pris ${ }^{19}$.

Remarquons au passage que SLT, dans son explication linguistique qui se veut objective, " oublie » de parler du troisième terme, la quasi homonymie Tansi/Tam'si, comme s'il voulait nous faire oublier qu'il reste évidemment commun aux deux noms. Or le pays, c'est justement le point aveugle sur lequel porte justement le malentendu. Car il y a Congo et Kongo. C'est la fameuse histoire de "la lettre volée ", qu'on ne voit pas tant elle est évidente, et surtout qu'on n'entend pas. D'où le malentendu.

\section{Le Congo, une passion ambiguë}

18 Côté Tchicaya, les choses sont assez claires, sa "passion " pour le pays a été maintes fois soulignée par des témoins et des confidents, même si le nom Congo peut prêter à confusion, à cause de l'homonymie entre le pays et le fleuve :

En 1956, le Congo est déjà «la pierre angulaire, le point de référence » de son œuvre : "Comme j'étais déçu par la maigreur de la Seine, je rêvais du Congo. » Et d'ajouter: «De même que mes aînés rêvaient de Négritude, je m'enchantais du Congo. $»^{20}$

19 Cette déception devant la Seine parisienne, source de nostalgie inguérissable et de rêve de retour, est un sentiment partagé par les deux écrivains. On trouve l'anecdote plusieurs fois relatée par Sony, notamment ici même, lorsqu'il répond à la question de savoir où il situe sa parenté « tropicale » avec l'Amérique latine :

Ils me disent : qu'est-ce que tu veux visiter ? Je dis : « Versailles et la Seine ». [...] J'ai dit que nous avons chanté au CM2 « la Seine, la Garonne, La Loire, le Rhône et la Seine arrosent toute la France ». Ce sont les grands fleuves français. Alors je me dis : si la Seine est un grand fleuve, le Congo doit être petit. On me dit: «la Seine, c'est ça ». Donc, à propos de la parenté géographique, moi, j'étais touché. J'ai dit : « c'est ça, la Seine? ça!» (rire). Alors quand je dis fleuve, je suis obligé de me méfier un 
peu. Sûrement, quelque part, je suis plus proche du point de l'Amérique Latine, parce que l'Amazone, c'est vaste, c'est aussi vaste... ${ }^{21}$ qu'on appellerait à présent la «littérature monde » et la tentation de repli dans le terroir local, sur le pays vili, comme dans cet entretien :

Comme je parle de moi, je dois récupérer tout mon savoir, tout ce que mon subconscient habite, c'est-à-dire, non pas toute la culture africaine, mais celle d'un homme né en pays vili au Congo. Ma passion est d'abord congolaise. [...]

On ne peut s'empêcher de penser à ce que nous disait Sony à Brazzaville : «Il manque des martyrs chez nous. Nous n'avons peut-être pas été assez capables de mourir pour notre pays. »

Cela vient du fait culturel ambiant. Plus personne ne connaît la culture de son pays et donc personne n'accepterait de se battre à mort pour sa défense. [...] Il faut avoir sa fierté d'être. Sony a raison et c'est malheureusement la grande affaire de ce continent dont la situation politique est déplorable. Au niveau de la vie de la cité, c'est le quant à soi ! [...] Bien sûr, mais ne parlons pas de l'Afrique, rien ne m'y autorise, je ne parle que de ce que je connais, c'est-à-dire ma région, où j'ai passé les quinze premières années de ma vie ${ }^{22}$.

Un observateur extérieur note avec perspicacité ces variations d'échelle dans la référence constante au pays, un territoire à référence flottante :

Il voulait parfois qu'on l'admette pour tel : un écrivain tout court. Ce qu'il était. Même s'il se voulait aussi « un Kongo " comme il le disait; et violemment, avec beaucoup de pudeur. Le pays, sa passion pour Loango, faisaient partie des choses qu'il taisait. On les devinait plus importantes que le reste. On sentait que ces choses restaient politiquement compliquées, affectivement douloureuses ${ }^{23}$.

Ainsi, s'il représente sans doute la pierre angulaire la relation entre Tam'si et Tansi, le Congo en constitue aussi bien la pierre d'achoppement. Car, lorsque Matondo note : « Il aimait son Congo qu'il voyait souffrir; il souffrait d'autant plus avec lui qu'il en était séparé physiquement ${ }^{24}$ », le possessif pose le problème de la référence. À chacun son Congo?

Encore une fois, c'est le cadet qui pointe franchement la différence de vision, le différend, au détour de sa réponse à la question sur ses liens avec son aîné :

D'abord un lien affectif, émotif, de départ. Tchicaya, c'était un vagabond, qui n'avait pas de pays. Tous les pays étaient son pays. Et il aimait de manière féroce le Congo. Enfin le Congo... Je ne sais pas lequel, parce qu'en plus il est allé au Congo belge... Lumumba n'avait aucune idée du Congo. L'idée que Lumumba avait du Congo lui avait été soufflée par les Belges, donc ça ne m'intéresse pas. Mais Tchicaya disait que chez nous, «le prince, c'est le fleuve ». Pour lui, il n'y avait pas Mobutu, il n'y avait pas Sassou, il n'y avait personne. C'est le fleuve qui gouvernait et qui commandait. Ça, c'était magnifique ${ }^{25}$.

Ces lettres n'ont malheureusement pas été retrouvées. Il n'est pas sûr que le fleuve, le mythe du fleuve trait d'union, suffise à les réconcilier. Car il a lui-même deux directions opposées, un amont et un aval ; un fleuve se remonte jusqu'à la source ou se descend jusqu'à la mer. Deux dynamiques symboliques que l'aîné et le cadet empruntent en des trajectoires souvent opposées, celui-là vers la mer du Loango, celui-ci vers la source du royaume du Kongo. Et on peut aussi observer des parcours contraires (et contrariés) même dans les genres qu'ils ont pratiqués l'un et l'autre, par lesquels ils ont été reconnus : pour TUT, d'abord la poésie puis le roman, à l'inverse de SLT dont les seuls recueils poétiques l'ont été à titre posthume. D'où cette impression, chez les deux, de 
faire partie d'une "génération sacrifiée » et mal aimée, comme le remarque justement B. Mongo Mboussa ${ }^{26}$.

\section{Sur l'autre rive, Kin qui rit, Kin qui pleure}

Bel exemple de ce chassé-croisé sur le fleuve: en 1984, TUT et SLT se retrouvent associés dans une revue parisienne pour donner leurs impressions sur « les capitales de la couleur ", dont Kinshasa est sans doute l'exemplaire le plus coloré. Autant la version de Sony est joyeuse, chaleureuse, «tropicale », mais passéiste, marquée par la belle et grande époque, à présent révolue, de la musique congolaise :

Malgré ses quelques géants de béton qui regardent le majestueux fleuve Congo et donnent l'image risible de la grenouille qui veut se faire aussi gros que la vache, Kinshasa n'a pas son cœur dans le béton et le néon. [...] Cette ville a son cœur à Matonge. Toute sa chair et tout son sang, sa sueur, ses odeurs, et surtout son sexe fait de musique et d'interminables rumbas; le tonnerre comme dit la fille, la foudre apprise, apprivoisée, la respiration " pieuvrosoïque ». [...] Cette ville est une longue chanson, une longue présence de Tabu Ley et du tout-puissant Lwambo Makiadi. Le premier pourrait être Verlaine et Rimbaud réunis, le second La Rochefoucauld, La Fontaine, La Bruyère et Molière. Je sais bien que cette référence est ridicule ${ }^{27}$.

Autant celle de TUT est pessimiste, critiquant tout rêve de retour en arrière ; son point de vue suit le cours du fleuve, tournée vers le cours du temps à venir, pressentant avec lucidité les défis qui attendent la ville de l'avenir :

C'est qu'il y a une vision romantique du village de l'enfance qui est considéré, à tort à mon avis, comme un paradis perdu. Et la ville n'est pas plus un paradis à conquérir comme l'espèrent ceux qui quittent volontairement aujourd'hui les campagnes. Ceux-ci se retrouvent vite en situation de conflit, embrigadés pour jouer du coude, jouer même de la révolution. Le rêve est fini. [...]

$\mathrm{Au}$ fond, le mal est déjà fait. Nous allons perdre toute l'expérience de chacun des groupes ethniques qui habitent tel ou tel pays, parce qu'il va produire un espèce de nivellement. C'est dommage, mais on ne peut pas revenir en arrière. Ce retour aurait pu se faire il y a vingt ans. Mais maintenant, les gens ont trouvé dans leur nouvelle situation des raisons d'être beaucoup plus importantes que dans ce retour en arrière que certains voudraient leur proposer, même s'il s'agit d'un retour en arrière ! [...]

Car la ville, si elle continue à regarder vers l'extérieur, va connaître des problèmes de plus en plus explosifs. Elle va davantage s'isoler, devenir un îlot, régresser et retourner à l'état de factorerie qui était le sien il y a un siècle et demi. C'est-à-dire l'endroit où l'on vient vendre la verroterie. Si elle veut cesser d'être le lieu de vente de la pacotille, si elle veut devenir un réel lieu d'échange, de commerce, de convivialité réciproque, il faut absolument qu'elle dynamise le marché intérieur.

Il n'existe pas de projet culturel dûment exprimé, pas de création de théâtres, ni de salles de spectacle. Quand il y a une salle de cinéma, ce n'est même pas une volonté endogène, c'est presque, ma foi, le mercantilisme de l'extérieur, pour diffuser quoi ? De la pacotille, des sous-séries B!

En dépit de cela, il y a des tentatives, presque comme des générations spontanées qui sont issues des mélanges de tout un tas d'éléments. La musique congolaise, par exemple, est le phénomène culturel le plus signifiant qui est apparu avec le développement des villes de ce pays, et qui a pris toute son ampleur avec l'arrivée de la radio et du disque. Ce phénomène a une double vocation. D'abord, montrer l'avènement d'une nouvelle musique d'essence syncrétique et de portée internationale. Ensuite, elle va être l'instrument de diffusion du lingala, une des langues du Congo, sous la forme syncrétique, elle aussi, du « lingua-franka ». [...] 
La créativité urbaine passe par la fluidité de circulation des gens et des biens. Sans cette fluidité, toutes les forces de propositions des individus nouveaux dont je parlais tout à l'heure resteront lettre morte. Et c'est alors la violence qui risque de triompher sur l'imagination ${ }^{28}$.

Pessimisme? prémonition? prophétisme? Peu importe, on est dans une vision de la modernité urbaine qui n'a rien perdu de son actualité.

\section{« Jusqu'à quelle profondeur du malentendu ? »}

La correspondance entre les frères congolais, le dialogue passionné qu'ils n'ont cessé d'entretenir, constitue sans doute la principale vie ultérieure de Tchicaya, ainsi que Sony le mentionne :

On s'est écrit longtemps avant qu'on ne se voie, en 1973, je crois. On s'est écrit, beaucoup, et dans ses lettres - je les ai - il y a des choses extraordinaires ${ }^{29}$.

Malheureusement, de ces lettres de Tchicaya à Sony, comme celles de Sony à Tchicaya d'ailleurs, aucune, à ma connaissance, ne nous est parvenue. Sauf la dernière sans doute, qui a été écrite à Paris deux mois avant que Tchicaya ne casse sa plume, et publiée à Brazzaville à l'occasion du dixième anniversaire de sa disparition. Dernière et unique, à ce double titre, à la fois testament d'amitié franche et témoignage personnel, il vaut la peine de la citer in extenso, parce que ces derniers mots écrits de Tchicaya à Sony (mais aussi ceux de celui-ci cités par celui-là) sont tous révélateurs du malentendu tragique qui aura marqué cette longue amitié littéraire :

Sony,

Depuis le retour de la Guadeloupe, je me promettais de t'écrire, n'ayant pas réussi à avoir une conversation avec toi, pendant dix jours passés dans la même ville. Ta carte que je viens de recevoir, a anéanti mes hésitations, mais me laisse bien pantois. Ce que tu dis est singulièrement confondant. C'est, ou frappé au coin de l'humour le plus sombre, ou de la sournoiserie la plus tragique. Je te cite: "Salut Gérald. De toute manière je suis celui qui est condamné à faire signe aux autres à n'importe quelle profondeur du malentendu... Je t'aime que tu le veuilles ou pas. » Voilà qui est surprenant. Peux-tu me dire ce qui t'a empêché de me faire signe à Basse-Terre, à Pointe-à-Pitre ? Toi, si bon prince tu proclames : « Je t'aimes que tu le veuilles ou pas. » Pourquoi offrais-tu ce masque hideux d'agacement et souvent de mépris?

Tu me permettras de douter, une fois de plus de ta sincérité à mon égard. Une preuve? Ton propos se veut magnanime? «Je suis celui qui est condamné... » Qui est le juge qui a prononcé à ton endroit une telle peine? "...à faire signe aux autres...» Qui sont ces autres? Sont-ils donc légion? «...n'importe quelle profondeur du malentendu. »Ce malentendu viendrait-il de ce que je pense pas que Shakespeare eût applaudi à Moi, Veuve de l'Empire? Par sympathie, je t'ai réservé la primeur de mes critiques sur cette pièce, dont l'écriture, la mise en scène laissent à désirer. Je confesse que je me suis ému de ce que tu prennes avec tant de désinvolture le parti de bâcler ton travail, est-ce là la source du malentendu? Possible. Eh bien tant pis! Je t'estimais assez pour ne pas te flatter bassement. Crois-moi, tout talent est fragile qui refuse le travail.

Je ne jubile pas quand tu écris : "Je t'aime que tu le veuilles ou pas. » Parce que j'en crois rien. On respecte les gens qu'on aime. Or tu n'as pas eu un seul signe d'amitié qui eût pu rendre le séjour à la Guadeloupe supportable. Tout le contraire. Nous étions trois écrivains congolais ${ }^{30}$ à la Guadeloupe, parce que nous n'avons pas eu un seul instant à être ensemble, en conciliabule, à faire le point, à boire un verre, ce voyage quoi qu'en en dise a été un fiasco. N'as-tu pas plus été préoccupé par la bagatelle que par ton travail ? Tu t'es laissé berner au point de mal gérer le crédit 
que t'avait ouvert ceux qui t'avaient invité ? Quel gâchis! Ce n'est pas par humeur, que j'en suis parti avant la date prévue. Je devais même en partir plus tôt... après la rencontre de Basse-Terre. Je préfère quant à moi ma solitude aux eaux troubles des rencontres manquées.

Mais, au fait, c'est sans doute une carte de vœux que tu voulais m'envoyer. Des vœux, je n'en lis nulle part. Ils te seraient restés sur le cœur, peut-être ? Voilà qui en dirait long, sur la spontanéité de ta sincérité.

Voilà cependant les vœux que je forme pour toi : Que le nouvel an te fasse plus économe de ta générosité, te fasse meilleur gestionnaire de tes talents multiples et te préserve des pires déconvenues. Si tu doutes de la sincérité de ces vœux-là, ma déception sera encore plus grande. Paix et bonne santé à ta famille, à toi. Tchicaya U Tam'Si ${ }^{31}$

Bien au-delà de l'anecdote sur les tempéraments de ce « couple infernal » (« rugueux » $v s$ « fougueux »), il est remarquable que le malentendu tourne autour de la question du théâtre, et plus précisément celui de Shakespeare, qui est LA référence commune aux deux dramaturges. Normal qu'elle soit donc aussi le terrain de leur désaccord. Côté Tchicaya, Sylvain Bemba, le père de la phratrie, avait déjà noté l'aspect et le ton « chakaspearien » de son théâtre :

Patrick de Rosbo, qui a préfacé Le Zulu, compare le héros de cette pièce à un «Macbeth noir» qui finit par être "découronné comme les maudits de Shakespeare ». Inévitable semble la référence au célèbre dramaturge, et bien réelles les analogies que l'on peut relever entre l'œuvre de celui que Neruda qualifiait de " plus vaste des êtres humains » et toute écriture théâtrale poétisée, surtout quand ses personnages sont eux aussi taillés dans «l'étoffe dont on fait les rêves ». De ce point de vue, Le Zulu est en effet... « chakaspearien », tout comme le Maréchal Nnikon Nniku, mélange de violence bouffonnante et de dérision amère ${ }^{32}$.

31 Côté Sony, la référence shakespearienne est encore plus nette, puisque la pièce en question, Moi Veuve de l'Empire, est explicitement tirée, adaptée du grand maître anglais. Ce qui n'empêche pas Sony, bien au contraire, de tirer la couverture shakespearienne à lui, vers son tropisme prophétique. Pour imaginer combien celui-ci a sans doute déplu à Tchicaya, il suffit de relire la dernière tirade de la pièce que Sony attribue bien sûr à Cléopâtre, de sorte qu'à cette nouvelle Kimpa Vita revienne le dernier mot :

Ouvrez mes intestins / donnez-les à manger à tous les chiens de la terre (...) Quoi / Vous voudriez que pour / vous dérober à votre couardise / je me change prophète / femelle? (...) Parce que l'Histoire reste fermée aux Nègres / Dites à Navès votre maître / qu'il est un navet / que ma bouche ne mangera pas / Dites-lui / qu'il est des femmes qu'on ne marchande pas / Cléopâtre jamais ne sera / réduite au rang / de femme usée / Elle sera libre (...) La guerre est finie / La conscience commence / Nous allons ouvrir l'Histoire / à tous les hommes. (Fin) ${ }^{33}$

\section{À chacun son Tchicaya ? Vers de nouvelles vies ultérieures...}

Revenons, pour finir, sur «Tchicaya passion ", où l'on découvre quatre lectures qui ont nourri l'écriture de ces jeunes écrivains. Des lectures diverses émergeant de la nouvelle génération congolaise, presque incomparables, en des contrastes équilibrés : deux voix féminines et deux masculines, deux habitées par le Congo (Ibéa Atondi, Wilfried Nsondé), deux habitant le Congo (Bill Kouelany, Dieudonné Niangouna); chacune tirant de la matière Tchicaya une manière différente, mais toujours critique. La pensée de l'«à demi » (Atondi), le maître du récit noir (Nsondé), l'apprentissage du comment 
vivre-voyou (Kouélany), et du comment «tourner le dos au cimetière » (Niangouna) ${ }^{34}$. Notons tout de même une constante remarquable : le nom du Congo n'y est pas écrit une seule fois... Faudrait-il s'en émouvoir ? Au contraire, à mon sens, c'est le symptôme que Tchicaya ne parle pas seulement pour son pays, qu'il reste moderne partout et pour tous, et représente encore, non pas la source vers où l'on part en pèlerinage, mais la mer où les jeunes feuilles vont se baigner, se perdre peut-être... Comme si ces quatre petites feuilles s'étaient donné le même mot d'ordre que Tchicaya, fidèles à son infidélité :

Le placenta, première place d'armes / jeté / Le cordon ombilical, lien premier / jeté / À quel abus de fidélité m'abonner. [...] Toute idée reçue est névrotique. Qu'importe d'où viennent le vent et le vin ${ }^{35}$.

Il n'y aura pas eu de Tchicaya reçu. Ni bantou, ni prophète, ni père, ni même frère..., il n'aura eu de cesse de chercher la reconnaissance de l'ami, du lecteur comme ami :

Si j'écris, c'est pour échapper réellement à une certaine solitude et pour essayer d'attirer le regard de l'autre. Le regard reconnaissant le plus large du terme. [...] Non pas que je me complaise dans cette solitude, mais je voudrais la briser. Briser ce cercle qui continue à se refermer sur moi [...] Je cherche simplement à sortir de ma solitude et mes livres sont des mains tendues vers ceux, quels qu'ils soient, qui sont susceptibles de devenir des amis ${ }^{36}$.

\section{NOTES}

1. Cultures Sud, $\mathrm{n}^{\circ} 171$, octobre-décembre 2008.

2. Voir mon « Prophète malgré lui », ibid., p. 89-95.

3. Cultures Sud, $\mathrm{n}^{\circ} 171$, octobre-décembre 2008, p. 7.

4. Ibidem, p. 114.

5. Ibidem, p. 42.

6. Ibidem, p. 110.

7. À noter que l'autre coordinateur scientifique, Daniel Delas, ne figure pas comme signataire, comme s'il avait fallu préserver l'équilibre correctement « congaulois ».

8. « Le poète et son pays intérieur. Entretien avec Jean-Baptiste Tati Loutard », Ibidem, p. 19.

9. « Témoignage d'Arlette Chemain », Ibidem, p. 110.

10. Pour une étude plus fouillée de leur itinéraires parallèles et croisés, voir mon «(Tchicaya) Tam'si et (Sony Labou) Tansi : même pays, même mer? ", dans Nino Chiappano (dir.), Tchicaya notre ami, Paris, Unesco, 1998, p. 45-62.

11. Entretien avec M. Kadima Nzuji, à Bayardelle, 1988, en partie reproduit dans Sony Labou Tansi, L'Autre monde, écrits inédits, Paris, Revue Noire, 1997, p. 150.

12. Lettre à Françoise Ligier, 30 septembre 1977. L'Atelier de Sony Labou Tansi. Correspondance I, Paris, Revue Noire, 2005, p. 212.

13. Lettre à José Pivin, Correspondance, op. cit., p. 84.

14. Lettre à Françoise Ligier, Correspondance, op. cit., p. 194.

15. Interview de Sony Lab'ou Tansi, propos recueillis par Françoise Ligier, décembre 1982.

16. « Donner du souffle au temps et polariser l'espace ", Recherche, pédagogie et culture, $\mathrm{n}^{\circ} 61,1983$.

17. « Le Pays intérieur du Prince Tchicaya U Tam'si », L'Afrique littéraire, $n^{\circ} 87,2^{\mathrm{e}}$ trimestre 1995. 
18. Sony Labou Tansi, « Lettre Infernale », dans Revue Noire, mars-avril 1993.

19. Entretien avec Djibril Diallo, 2 juin 1987, dans Sony Labou Tansi. Le sens du désordre, textes réunis par J.-C. Blachère, Montpellier, Université Paul Valéry, 2001, p. 173-181 (p. 173).

20. J.-B. Tati-Loutard, « Oraison funèbre », Europe, octobre 1991, p. 143.

21. Entretien à Bayardelle, 1988, inédit.

22. A. Brezault et Gérard Clavreuil, Conversations congolaises, L'Harmattan, 1989, p. 107-125.

23. Jean-Maurice de Montremy, « Souvenirs », Europe, octobre 1991, p. 147.

24. Matondo Kubu Turé, "Mise en scène d'une poésie, mise en verbe d'une écriture ", Europe, octobre 1991, p. 129.

25. Entretien avec Bernard Magnier, 26 octobre 1993, dans Sony Labou Tansi. Paroles inédites, Éditions Théâtrales, 2005, p. 55.

26. Tchicaya passsion, op. cit., p. 97-100.

27. "Le sexe de Matonge", dans Capitales de la couleur (Bruno Tilliette ed.), Autrement, 1984, p. 258.

28. «La marmite de sorcière et l'homme nouveau », dans Capitales..., op. cit., p. 308-311.

29. Entretien avec Bernard Magnier, op. cit., p. 55.

30. Le troisième étant Maxime Ndébéka.

31. Lemba, bi-mensuel culturel publié par l'Association Sony Labou Tansi pour la Culture, $\mathrm{n}^{\circ} 001$ du vendredi 15 mai 1998, p. 5.

32. Sylvain Bemba, « Un théâtre de la passion et des passions ", Europe, octobre 1991, p. 121, 125.

33. « Moi, Veuve de l'Empire », L'Avant-Scène, 1987, p. 11, 15 et 32.

34. Tchicaya passion, op. cit., respectivement : «Venir après », p. 107-108; « La puissance du verbe tchicayen ", p. 115 ; «Comme un signe de mauvais sang », p. 113 ; «De la bibliothèque familiale aux gorges de Diosse », p. 114.

35. «La Source », Revue Noire, $n^{\circ} 5$, juin-juillet-août 1992, p. VI-VII.

Publié à titre posthume, ce texte est contemporain de la dernière lettre à Sony auquel il paraît apporter une réplique sur le mode hybride de l'essai et du poème, comme l'indique en introduction sa destinataire, Nicole de Pontcharra : «"Je préfère courir au vent de l'histoire ou me saouler de vin, c'est une revanche à prendre sur l'agonie qui viendra toujours trop tôt. Trêve de fatalisme." Ces quelques lignes prémonitoires accompagnaient les vœux de bonne année et le texte sur La Source que Tchichaya m'adressait au début de l'année 1988. »

36. Alain Brezault et Gérard Clavreuil, Conversations congolaises, Paris, L'Harmattan, 1989, p. 107-125.

INDEX

Mots-clés : Congo, Tchicaya U Tam'si, Sony Labou Tansi, poésie congolaise, Rimbaud

\section{AUTEUR}

\section{NICOLAS MARTIN-GRANEL}

NICOLAS MARTIN-GRANEL est agrégé de Lettres Classiques et diplômé de Sciences Politiques.

Enseignant chercheur dans diverses universités africaines, il a publié des ouvrages anthologiques 
et nombre d'articles critiques sur la littérature africaine, notamment congolaise. Ses recherches actuelles en génétique textuelle portent sur l'oeuvre et les manuscrits de Sony Labou Tansi dont il est l'éditeur scientifique (L'Autre Monde, L'Atelier de Sony Labou Tansi, Revue Noire, 1997 et 2005, Poèmes, CNRS Éditions, 2015). Membre du comité de rédaction des revues Cahiers d'Études Africaines et Riveneuve Continents, il est rédacteur en chef des Études Littéraires Africaines et chercheur associé à l'ITEM-ENS-CNRS dans l'équipe Congo / Manuscrits Francophones. 